\title{
Fair Partnerships - Working with NGOs
}

\author{
Shikoh Gitau and Gary Marsden \\ ICT4D Research Centre, University of Cape Town, South Africa \\ \{sgitau,gaz\}@es.uct.ac.za
}

\begin{abstract}
This paper highlights how Non-Governmental Organizations (NGOs) can be utilized during the design of Information Communication Technologies for Development (ICT4D). We use the design process of a voter education system as a case study, which incorporated three NGOs from two African countries. Of key interest to us are the ways in which we can avoid exploiting these NGOs and make sure the ICT intervention meets their goals, as well as those of the researchers.
\end{abstract}

Keywords: Public Display, ICT4D, Participatory Design, Contextual Design, Mobile Phones, Africa, developing world, NGOs.

\section{Introduction}

Some of the challenges facing the design of technology relevant for developing community include: understanding the users in their context; opportunity identification; determining design requirements as well as evaluating the impact caused by the resulting technology. These are mainly caused by differences between the researcher (mostly coming from a developed context) and the community of interest; the differences can be in terms of language, culture, attitudes and locality (where designers and users are separated geographically during some stages in the design process). These disconnects have had costly effects in ICT4D projects, with close to $70 \%$ of all initiated projects failing within the first few years of conception [1]. Hence there is a need for an alternative means to approach the design of ICT4D.

Non-Governmental Organizations (NGOs) are an instrumental and integral part in Africa's development agenda; they exist as non-profit citizen groups, organized on a local, national or international level. NGOs have enjoyed high levels of trust and acceptance in developing communities, and are now used to provide information, analysis and expertise about these communities. They also help with the implementation of government projects and monitoring of agreements and policies. Using an NGO for community liaison is therefore an attractive prospect for ICT researchers wishing to create technologies for developing world communities, and reports of such partnerships are common, e.g. [2]. However, instead of using the NGO just as a liaison element in development projects, with roles limited to 'access point' or 'contact', we believe that it is important for these NGOs to be incorporated in a more integral role both in the development and evaluation processes for the project as they will remain in the community long after the technologist or researchers have left. We believe this type of evaluation mechanism is absolutely critical in working with an 
$\mathrm{NGO}$ as it will allow them to measure in their own terms any improvements the ICT intervention has made and determine what needs to be done to afford more impact.

In the rest of this paper we will report on working with three NGOs in the domain of voter education in Africa as part of a project to create new forms of multimedia voter education material. Voter education (VE) is a term used to refer to the process of disseminating materials and programmes designed to inform the electorate about the specifics and mechanics of the voting process for a particular election and aimed to enlighten them or convince them to participate in the election.

Three NGOs participated in this study, all of whom are involved in democracy and governance issues in Africa; specifically Kenya and South Africa. In Kenya we worked with the Centre for Multiparty Democracy-Kenya and Media Focus on Africa. In South Africa we worked with the Institute for Democracy in South Africa.

\section{Methodology}

Before the design process could begin, we needed to understand the voter education process from the NGO's perspective and context, as well as conduct document ethnography where we followed the voter information development process. We first sent out an initial questionnaire to the NGOs whose aims were to capture basic demographic information as well as the technical competency of their staff. This information allowed us to plan our study such that it would suit the NGO's schedules and technical abilities. Thereafter, we carried out a contextual inquiry study, where we took an 'apprentice mode' within the communication office that is in charge of the voter education process.

A contextual inquiry allows the designer to learn more about the user by observing them in their daily routine; this is especially important in circumstances where the designer has little or no understanding of the user's domain. We therefore observed the NGO staff as they carried out their day to day tasks throughout the voter education process in addition other tasks within their mandate. We followed these observations with unstructured interviews to clarify some of the observations that were made but were not explicit. This triangulation of techniques allowed us to capture information that we had missed previously and clarify observations made and inquire deeply into given actions.

We made notes for both the observation sessions as well as the interviews and digital photos were also taken of some the key moments.

\section{Findings}

The study found that voter education is a very complex but deliberate process that went beyond the mere dissemination of voter information material. We noted that each piece of information - the message - was tailored towards a particular segment of voters according to their demographic data. Voter education messages were disseminated either directly through face to face encounters (such as workshops), door to door visits or through broadcast media including print media, television, and radio. The radio was the dominant media, taking over $50 \%$ of the total VE budget dues to its wide spread use in both these countries. 
NGOs monitored the impact of the VE process through a fortnightly survey, whose result determined whether a message achieved its intent, whether it should continue running or if it should be changed for a different segment of voters or moved to a different media.

During the study, we introduced the Big Board (BB)[3] as complimentary media for voter education that allowed sharing of multimedia using large public displays and mobiles phones via Bluetooth transfer. Using a participatory design approach, we designed a PC based application, that would allow the NGO staff to create, manipulate and upload media onto the $\mathrm{BB}$, that will then be accessed by voters on their mobile phones. This media could be adjusted and cycled to fit the various voters needs, through a simple drag and drop interface (a technique familiar to the NGO staff). The application, as envisioned by the NGO would facilitate in cutting down on operational cost incurred from outsourced services but still enable a widespread reach via mobile phones with the assumed viral spread of the messages. Through various iterations, observations and task based analysis we ensured that the NGO staff could comfortably use this application to upload media onto the BB.

The cooperation with NGOs during the study not only ensured that we could develop appropriate solutions for information dissemination by providing us with an understanding of the voters and voter education processes; but their ability to measure the envisoned impact (through their standard monitoring tools) allowed them and us to have the confidence that they could evaluate the effectiveness of the BB system during a voter education process.

\section{Discussion}

This is a research that, if we had attempted to do on our own, would certainly have led to failure. We discovered that voter education turned out to be more complex than we had initially envisioned, where demographics, illiteracy, trust and integrity are critical aspects that are largely opaque to the outsider. Furthermore, we did not have the capacity in terms of staff and funding to properly explore these issues to a point where we could make a contribution.

So, once again we see the value of having a 'human-access point' into the community [4]. We could leverage their deep understanding of the people and their needs to help create solutions in developing community settings. What is less clear, however, is what the NGO gets out of their collaboration with us. If we are not to be accused of propagating imperialism in Africa once more (this time in the form of culture or technology) then joint research of this nature must be an equal partnership. From our experience we propose that this equality should not be in the form of task-sharing (it is pointless for the NGO to learn how to write software, just as it would be for us to learn all the laws relating to the conduct of elections). Instead, we recommend that each party have in place a meaningful evaluation method that can be applied at the end intervention. In this way, we believe that the research partnership can be kept 'honest' as there is a clear understand of what constitutes success for each party. 


\section{Conclusion}

Ostensibly, this research is about the creation of a voter education system in Africa. A wider learning in the field of HCI4D (for the lack of a better term) is the way in which NGOs should be engaged in this type of research partnership. Many papers report on the data and advantages of working with established NGOs and human-access points ([4] for example) but do not report on how the NGO feels about the intervention. However, in at least one case we are aware of, an NGO partner was unhappy at the intervention.

Our experience suggests that these problems can be avoided by making sure the NGO has an evaluation method in place that can inform them of the impact of your intervention in their domain. In fact, we would argue that such a partnership must not be entered into until such evaluation methods exist. By this token, we can ensure equal benefit to both the research community and the NGO.

Acknowledgments. We would like to thank the three NGOs involved in this work for the patient support of our blundering attempts. We would also like to thank Microsoft Research Cambridge who funded the research.

\section{References}

1. Heeks, R.: Information Systems and Developing Countries: Failure, Success, and Local Improvisations. The Information Society 18(2), 101-112 (2002)

2. Chetty, M., \& Grinter, R. HCI4D: HCI Challenges. In: Proceedings CHI 2007, pp. 2327 2332 (2007)

3. Maunder, A., Marsden, G., Harper, R.: SnapAndGrab - Accessing and sharing contextual multi-media content using Bluetooth enabled cameraphones and large situated displays. In: Proceedings CHI 2008, pp. 2319-2323 (2008)

4. Marsden, G.: Towards Empowered Design. IEEE Computer 41(6), 42-47 (2008) 